\title{
Saúde Coletiva: um desafio para a enfermagem
}

\author{
Collective health: a challenge for nursing
}

Silvia Matumoto 1

Silvana Martins Mishima 2

Ione Carvalho Pinto 2

\footnotetext{
1 Secretaria Municipal de Saúde de Ribeirão Preto. Rua Patrocínio 2201, Ribeirão Preto, $S P$ 14085-530, Brasil. smatumoto@uol.com.br 2 Departamento de Enfermagem MaternoInfantil e Saúde Pública, Escola de Enfermagem de Ribeirão Preto, Universidade de São Paulo. Av. Bandeirantes 3900 , Ribeirão Preto, SP 14040-902, Brasil. smishima@glete.eerp.usp.br ionecarv@glete.eerp.usp.br
}

Abstract This study takes public health as the point of departure to discuss both the concept of collective health and its object and field of action, emphasizing the concepts of man, collective and individual spheres, and health-disease, through a discussion of the interdisciplinary construction of the collective health field. The authors then place nursing within this field, emphasizing what appears to be the greatest challenge, i.e., to approach nursing as a practice of relationships.

Key words Health; Public Health; Nursing

Resumo O texto parte da saúde pública para discutir o que é saúde coletiva, seu objeto e campo de atuação, dando ênfase à concepção de homem, à discussão a respeito do âmbito individual e coletivo e ao conceito de saúde-doença, passando pelo debate da construção interdisciplinar da saúde coletiva. Em seguida, busca situar a enfermagem nesse campo, destacando aquilo que parece ser um grande desafio, compreender a enfermagem como prática de relações.

Palavras-chave Saúde; Saúde Pública; Enfermagem 


\section{Introdução}

O debate a respeito do que é saúde coletiva, seu objeto e âmbito de atuação parece-nos pertinente e vem sendo trabalhado por alguns autores, como Felipe (1989), Canesqui (1995) e Nunes (1994). Esse campo vem sendo marcado por imprecisões, pela multiplicidade de possibilidades de definição, apresentando, como traço fundamental, o fato de estar em constituição, como conhecimento e prática, na sociedade brasileira.

Pretendemos efetuar uma aproximação e discussão das práticas em saúde coletiva que se manifestam no cotidiano das unidades de saúde, bem como situar a enfermagem nesse espaço, para compreender como esta tem-se constituído enquanto uma das disciplinas que constróem a saúde coletiva e qual a contribuição que traz para este coletivo. Neste sentido, aqui parece estar o grande desafio para a enfermagem: compreendê-la como prática de relações entre os homens, seus saberes e projetos.

\section{Uma aproximação ao campo da saúde coletiva}

Nossa abordagem ao termo saúde coletiva vem da circunvizinhança desta com a saúde pública, ou melhor, de seu surgimento dentro deste campo (Felipe, 1989; Nunes, 1994; Canesqui, 1995). A saúde pública conforma-se em um campo de saberes e práticas demarcado tradicionalmente pela intervenção técnica e política do Estado na assistência, a qual privilegia a "base técnica" do conhecimento científico, ancorado nas técnicas de investigação empíricas - em particular, na área bacteriológica -, cujas práticas voltam-se tanto para o individual como para o coletivo (Pires Filho, 1987). No primeiro caso, através de ações de caráter preventivo e de promoção à saúde com atividades de assistência médica e reabilitação; no segundo, desenvolvendo as ações governamentais das políticas de saúde dirigidas ao coletivo.

Na saúde pública, a ação de saúde representa o instrumento técnico-político que irá intervir no processo saúde-doença, quebrando sua cadeia causal mediante o tratamento e a reabilitação do indivíduo doente, ou evitando seus riscos e danos por intermédio da prevenção e promoção da saúde, além do controle dos sadios.

Em nosso cotidiano, essas ações apresentam-se, muitas vezes, na forma dos programas que se ocupam de alguns grupos de risco - tais como, crianças, gestantes, mulheres (mais recentemente) e idosos - ou para grupos acome- tidos por algum dano - como os hipertensos, as pessoas com tuberculose, com hanseníase, com diabetes, portadores do vírus HIV ou com AIDS. Ações de assistência médica individual diante de alguma circunstância pontual - como nas atividades de pronto atendimento configuram também um instrumento para intervir na cadeia causal da doença. O objeto destas ações é um homem genérico que, naquele momento ou circunstância, se enquadra segundo um risco ou dano dentro do processo saúde-doença.

Quanto às ações dirigidas ao coletivo, os exemplos que podem ser citados são as tradicionais campanhas de vacinação ou as ações de controle de doenças, como a dengue, a cólera, ou as ações de educação em saúde. Nesse "coletivo", o homem é tomado como um ser "em geral”, um conjunto homogêneo de pessoas, para o qual se dirige a ação frente ao risco ou dano comuns.

Em sua história, a saúde pública brasileira assumiu o coletivo sob o aspecto de algo exterior ao indivíduo, ou seja, a coletividade é referida enquanto uma coleção de indivíduos (Merhy, 1985). Nela, as intervenções em saúde também se dão externamente, isto é, sempre serão as mesmas, atingidas progressivamente em função da evolução linear e sucessiva do conhecimento científico relativo ao meio externo. Para ilustrar, podemos pensar a questão da AIDS, em que todo conhecimento produzido a respeito da forma de transmissão e da doença poderia, em tese, já tê-la colocado sob controle através do uso de camisinhas e do não compartilhamento de seringas. Contudo, parece que este olhar não considerou devidamente as relações da vida cotidiana entre os homens, ou seja, seus afetos, medos, incertezas, a sexualidade e outros, que a saúde coletiva tenta abordar.

Esta outra aproximação para a compreensão do coletivo significa apreendê-lo enquanto campo estruturado de prática sociais, "que como totalidade se tornaria ponto de partida para a compreensão e estruturação das práticas sanitárias" (Merhy, 1985:21), quando o próprio objetivo dessas práticas adquiriria historicidade. Dizer isto, significa reconhecer outra concepção de homem, o homem como um ser social, em constante relação com outros homens e com seu meio, transformando-o e sendo transformado por ele, isto é, um protagonista da ação de saúde que se constrói. Este homem é, ao mesmo tempo, sujeito, ator social, protagonista e objeto da ação, aquele que se submeterá às intervenções.

Segundo Pereira (1986), a aproximação da área das ciências sociais ao campo da medici- 
na social - que estendemos ao da saúde coletiva - contribuiu para entendê-la como “...uma ciência histórico-social, percebendo que as características dos seres humanos (doentes ou não) são sobretudo um produto de forças sociais mais profundas, ligadas a uma totalidade econômico-social que é preciso conhecer e compreender para explicarem-se adequadamente os fenômenos de saúde e de doença com os quais ela se defronta" (Pereira, 1986:30).

Seu objeto não é mais o corpo biológico, mas os corpos sociais. "Não se trata, tão-somente, de indivíduos, mas de sujeitos sociais, de grupos e classes sociais e de relações sociais referidas ao processo saúde-doença" (Pereira, 1986: 30). Trocando em miúdos, demarca-se o conceito saúde-doença da saúde coletiva com base na determinação social do processo saúdedoença, diferentemente daquele da saúde pública, da causalidade.

A saúde coletiva é um campo ainda em constituição no Brasil, assumindo diversas formas e abordagens (Canesqui, 1995). Esse termo surgiu no fim da década de 70, em um momento de reordenamento de um conjunto de práticas relacionadas à questão da saúde, diante da necessidade de outros saberes, além dos da medicina, para a compreensão do processo saúde-doença e da convivência cotidiana entre diferentes profissionais.

A demarcação aqui colocada é para a interdisciplinaridade. A saúde coletiva é um "campo de práticas diversas socialmente determinadas, que se apoiam em diferentes disciplinas científicas, numa zona de 'encontro de interdisciplinas"” (Felipe, 1989:63). Nesse "encontro" instaura-se uma dinâmica em que se fazem presentes contradições e conflitos de interesses entre os trabalhadores de diversas áreas de conhecimentos, sujeitos sociais, cada um com uma conformação específica para olhar o processo saúde-doença e as práticas a este concernentes.

Nunes (1994) reitera estas idéias ao colocar saúde coletiva constituída nos limites do biológico e do social, tendo como desafio "compreender e interpretar os determinantes da produção social das doenças e da organização social dos serviços de saúde" fundamentando-se na "interdisciplinaridade como possibilitadora da construção de um conhecimento ampliado da saúde e na multiprofissionalidade como forma de enfrentar a diversidade interna ao saber/ fazer das práticas sanitárias" (Nunes, 1994:19).

Isto explica, de certa forma, as diferenças na identificação do que é problema e o que pode ser uma intervenção, não somente do ponto de vista técnico-científico, mas no atendimento das necessidades expressas pela clientela, como, por exemplo, a situação de uma puérpera com problema de ingurgitamento mamário que se apresenta em uma unidade de saúde referindo "dor nas mamas". Esta conjuntura será vista e tomada de diferentes formas se o atendimento for dirigido por uma enfermeira, um auxiliar de enfermagem, um médico, um assistente social, uma psicóloga ou qualquer outro profissional, os quais, independente de sua formação específica, conformam olhares distintos em função das relações que estabelecem em sua vida cotidiana.

A construção dessa interdisciplinaridade dentro da "equipe de saúde" vai-se dando na identificação e respeito pelo núcleo de competência e responsabilidade de cada profissional, aquilo que é específico em termos de saberes e responsabilidades e pelo campo de competência e responsabilidade comuns a todos os trabalhadores de saúde (Campos, 1997).

Gomes \& Deslandes (1994) também defendem que, nesta construção, não se anula a disciplinaridade, "assim como não significa a justaposição de saberes também não anula a especificidade de cada campo de saber. Ela, antes de tudo, implica numa consciência dos limites e das potencialidades de cada campo de saber para que possa haver uma abertura em direção de um fazer coletivo" (Gomes \& Deslandes, 1994:111).

Complementando essas afirmações, ousamos utilizar uma idéia de Almeida Filho (1997) empregada ao discutir a transdisciplinaridade, sem nos preocuparmos, contudo, com a definição desse termo. Destacamos a idéia do autor, ao colocar a "possibilidade de comunicação não entre campos disciplinares, mas entre agentes em cada campo através da circulação não dos discursos (pela via da tradução), mas pelo trânsito dos sujeitos dos discursos" (Almeida Filho, 1997:17). Dizendo de outra forma, a conformação de uma prática de saúde a partir da convivência de saberes diversos e trabalhadores de diferentes áreas de formação, possibilitando olhar seu objeto, esse sujeito social, a partir de focos não coincidentes, dispostos pelos diferentes saberes e práticas que se transformam nessa mesma convivência.

Nesse sentido faz-se essencial entender a equipe de saúde como rede de relações tecida no cotidiano, entre agentes que portam saberes diferenciados e desenvolvem práticas distintas (Fortuna, 1999), tornando-se necessário certa disponibilidade para que estes reconheçam e respeitem tanto suas desigualdades no trabalho (Peduzzi, 1999) quanto os problemas que se apresentam na sociedade contemporânea: a iniquidade, a insegurança social e a deterioração do ambiente (Nunes, 1994), cons- 
truindo-se, a partir daí, um projeto de trabalho que instaure uma lógica ética e socialmente solidária.

\section{A enfermagem na construção da saúde coletiva}

A enfermagem, enquanto uma das disciplinas que compõem o trabalho em saúde, constituise também como uma daquelas que constróem a saúde coletiva.

- Como a enfermagem se insere nesse campo da saúde coletiva?

- Como o saber da enfermagem se relaciona com outros saberes que constróem a saúde coletiva?

- Como a enfermagem se faz enfermagem?

Retomando o que já foi explicitado anteriormente quanto à compreensão do coletivo como um campo estruturado de práticas sociais que caracterizam a saúde coletiva, é importante, no que se refere à discussão da enfermagem na construção deste campo, que nos aproximemos mais detidamente do conceito de prática social.

"Quando se fala em práticas sociais, e no caso a saúde ou a enfermagem, estamos nos referindo a tomar estas práticas para além de sua dimensão profissional e técnica, ou seja, para além de uma aplicação imediata e direta dos conhecimentos técnico científicos. Estamos nos referindo a tomar em consideração a dinâmica social ou seja, as inter-relações de cada âmbito de prática com as demais, seja na produção do conhecimento, na reprodução sócio-econômica e política e na inserção dos sujeitos" (Almeida et al., 1999:3).

Desta forma, na definição de práticas sociais, parte-se da categoria trabalho, considerando-as enquanto práticas constitutivas da sociedade (Schraiber, 1996). Estamos então tomando em conta que a enfermagem não se apresenta neutra e não se faz somente com saberes e práticas técnicas, ainda que necessite destes para constituir-se como um trabalho social em dada sociedade, reconhecido por ela e intervindo nela.

$\mathrm{Na}$ enfermagem, segundo Almeida et al. (1999), estudos nessa direção aparecem, no início da década de 80, com contribuições relevantes de Almeida et al. (1981), Germano (1983) e Silva (1986), disparando um processo de efervescência na produção de conhecimento em diferentes áreas da enfermagem e, em particular, da enfermagem em saúde coletiva.

A produção teórica da enfermagem em saúde coletiva vem-se conformando em estudos que se dedicam tanto às determinações mais gerais presentes nas macroestruturas quanto nas micro relações presentes no cotidiano do trabalho.

Almeida et al. (1999) destacam a contribuição teórica da enfermagem em diferentes áreas temáticas na saúde coletiva, como, por exemplo: o ensino na instrumentalização para a intervenção (Egry, 1996); a organização tecnológica do trabalho em situações específicas, tais como na hanseníase, na tuberculose e na assistência à mulher (Lana, 1992; Ferreira, 1994; Vaz, 1996); o processo histórico da prática de enfermagem em saúde pública (Villa, 1992); os processos organizativos voltados para a gerência e supervisão de serviços de saúde e de enfermagem (Mishima, 1995; Silva, 1997); os modelos de assistência de enfermagem em saúde coletiva (Corbishley, 1998); as transformações do trabalho em saúde coletiva na reestruturação do trabalho em saúde (Cocco, 1997); a dialética objetividade e subjetividade presente na micropolítica do trabalho em saúde, focando aspectos do acolhimento e das relações estabelecidas no trabalho de equipe em saúde (Matumoto, 1998; Fortuna, 1999; Peduzzi, 1999).

Mais recentemente, essas temáticas têm-se dirigido a uma análise da dimensão do sujeito da ação na intervenção em saúde, tanto sob o foco do usuário como dos trabalhadores de saúde, buscando enriquecer a ação cuidadora na perspectiva da integralidade da assistência à saúde.

Todas estas questões - e algumas mais apontam para o entendimento que hoje temos da enfermagem como uma prática de relações, como sempre foi e sempre será, que se utiliza de um saber tecnológico, construído desde Florence Nightingale, o qual se reformula e conforma intervenções distintas, em cada momento histórico, nesta convivência dos trabalhadores de saúde e de enfermagem, entre si e com os usuários, no cotidiano dos serviços de saúde inseridos em um contexto social.

A questão aqui colocada, parece situar-se em torno do objetivo/finalidade do trabalho de enfermagem. É o para quê se faz esse trabalho. A enfermagem está incluída na "equipe” para responder por uma parte da assistência, naquilo que lhe é específico, como o cuidar ou executar as ações decorrentes do trabalho médico, tais como aplicar medicações ou fazer curativos. Incluem-se ainda algumas questões do âmbito do trabalho de gerência da equipe de enfermagem feito pelo enfermeiro. Qual finalidade é dada a este trabalho? Quais intervenções são pensadas e colocadas em prática? Providenciar infra-estrutura material para a assis- 
tência acontecer? Fazer as escalas dos "recursos humanos" de enfermagem (aqui tomados como coisas)?

Destacamos estes aspectos para refletirmos a respeito de que projetos estamos construindo com nossas práticas de enfermagem, mais envolvidos com o fazer saúde que qualifique a vida?

Aqui está o nosso desafio.

Construirmos um projeto coerente com os princípios do SUS, de acesso e universalidade do direito à saúde, equidade e integralidade, respeitando o direito à cidadania. Um projeto no qual tomamos o homem - incluindo-nos nele - na qualidade de um ser constituído socialmente, um ator social que tem um projeto, uma intencionalidade no fazer, e que está em uma linha de produção de fatos sociais (Testa, 1995). O projeto de intervenção da enfermagem vai na direção da produção de uma ação social, a qual pode ser de manutenção ou de transformação da realidade.

Partimos da premissa de que as práticas de saúde determinam e são determinadas no cotidiano e, por isso, reiteramos que a enfermagem não é neutra. O simples fato de não se fazer nada ou de achar que não se tem nada a ver com o caso, já configura uma posição assumida, ou seja, a da manutenção, deixar as coisas como estão. Aqui retomamos o objetivo/finalidade do nosso projeto. Qual o impacto de nossas práticas sobre a saúde da população pela qual nos responsabilizamos?

Isso nos remete à especificidade da enfermagem nesta construção interdisciplinar da saúde coletiva, ou seja, a enfermagem deve assumir seu núcleo de competência e responsabilidade, o cuidado. Cuidado este que se volta para o doente e não para a doença - atravessando subjetividades individuais e coletivas - e que exige, para ser viabilizado, o uso de saberes oriundos de diversas disciplinas.

A enfermagem, tendo o cuidado como núcleo de competência e responsabilidade, manifesta potência para transitar em diferentes campos de conhecimento para a prestação deste cuidado, ou melhor, tendo como foco a pessoa a quem cuidará, a enfermagem pode estabelecer mais intensivamente canais de interlocução com agentes de outras disciplinas e, em conjunto, buscar tecnologias necessárias à assistência, estabelecendo relações com a equipe e com a família, atuando no processo de transformação da realidade. Neste sentido, consideramos que esta prática social apresenta grande possibilidade de contribuir para a construção de um saber interdisciplinar na saúde coletiva segundo a direção apontada por Al- meida Filho (1997), a de estabelecer trânsito dos "sujeitos dos discursos" para criar uma nova práxis.

Retomando a questão do projeto e das práticas que executamos e vivenciamos, nós nos produzimos enquanto homens, nos reconhecemos e nos fazemos reconhecidos pela sociedade. Uma prática que, ao se constituir, passa pelo mundo interno do homem, o trabalhador de saúde - primordialmente, um ser humano -, no sentido de torná-lo consciente de si e, ao mesmo tempo, fazendo com que possa ajudar o outro, com o qual se relaciona, a tornar-se consciente de si também.

Em nossas relações de trabalho temos que considerar o usuário enquanto homem constituído socialmente, da mesma forma que suas necessidades. O que são estas necessidades? Como se conformam? As que nos interessam aqui são as necessidades de saúde.

As necessidades do homem não são quaisquer: “(...) aparecem como aquilo que precisa 'necessariamente' ser satisfeito para que esse ser continue sendo um ser" (Mendes-Gonçalves, 1992:19) em dada sociedade. Tomando como exemplo as práticas dos xamãs, estas constituíam-se em uma forma de atender necessidades de saúde por meio de rituais, rezas e benzeções que mediavam as relações entre o homem e a natureza, para debelar o "mal" que determinava restrições à capacidade humana de viver a vida. O xamã era responsável - e assim reconhecido - pela execução deste trabalho que, provavelmente, tinha formas variáveis de sociedade para sociedade.

As práticas de saúde atuais lidam com a identificação de necessidades constituídas da mesma forma em processos sócio-históricos. Estas necessidades podem apresentar-se de diferentes formas - como, por exemplo, solicitação por consultas médicas, curativos, imunização, exames laboratoriais, radiológicos ou outro tipo qualquer de atenção do trabalhador de saúde -, as quais podem ou não ser reconhecidas e tomadas como objeto de trabalho. Nesta relação de reconhecimento de necessidades individuais e/ou coletivas, fazem-se presentes a concepção do processo saúde-doença e o projeto que se defende, considerando-se as necessidades a partir de um recorte biológico ou considerando-se as relações da vida em sociedade, transformando-as nesse filtro, objeto das ações de saúde.

As necessidades de saúde são também histórica e socialmente determinadas, bem como referidas a uma sociedade. Elas não se apresentam como necessidades de saúde em geral e devem ser satisfeitas de forma específica por pro- 
fissionais e instrumentais próprios, conforme o estabelecido pela sociedade em que se insere.

As práticas de saúde devem estar referidas a "necessidades sociais" que são aquelas caracterizadas como "sempre necessidades de indivíduos, que como são sempre de indivíduos sóciohistoricamente determinados, isto é, que estão em relações sócio-históricas com outros indivíduos, são produzidas por essas relações e para essas relações, sem deixarem nunca de serem individuais" (Mendes Gonçalves, 1992:20). Tais práticas comportam uma racionalidade com finalidade concreta, um projeto de ação; criam e consolidam um sistema de necessidades, cujo contorno é dado pela sociedade mediante valores e normas.

Neste momento, o SUS constitui uma das manifestações de um conjunto de "necessidades sociais" de saúde, as quais portam um caráter ético-moral que defende a saúde como direito de todo cidadão, propondo-se a garanti-lo através de seus princípios de acesso, universalidade, eqüidade e integralidade. É neste conjunto de necessidades que precisamos apreender os problemas dos usuários que atendemos e traçar o projeto para a enfermagem.

Todavia, o alcance da determinação das necessidades não está distante de nós, como pode parecer. Destacamos aqui nosso papel de geradores de necessidades, lembrando como nossa prática atual - do pronto atendimento - atua como geradora de necessidade de mais e mais consultas, na medida em que só vislumbramos e oferecemos consultas médicas como solução aos problemas de saúde que nos aportam.

É preciso duvidar das soluções mais óbvias para os casos, lembrando sempre que nossa apreensão da realidade é sempre parcial e a partir de um ponto de vista. Nosso desafio, o da enfermagem, é quebrar este olhar cristalizado dentro de nós, para abrirmos o horizonte de possibilidades de apreensão do que é saúdedoença e do que pode ser alternativa de solução para os problemas que se nos apresentam.

Observamos também que isto hoje já é feito assim, mas somente para alguns casos; aqueles que nos interessam por algum motivo ou interessam a alguém mais próximo de nós. Referimo-nos àquelas situações em que marcamos uma consulta a mais, articulamos atendimentos entre áreas e serviços diversos, entramos em contato com outros trabalhadores para acelerar algum atendimento ou para apontar a preocupação principal deste, e muitas outras. Se, nesses casos, quebramos a norma, a rotina, o estabelecido, por que não mudar o foco do trabalho também no tratamento do usuário "em geral" e utilizar todas as possibilidades e recursos disponíveis, de modo a ultrapassar o âmbito do biológico e considerar as relações sociais na vida, para pensar as possibilidades de soluções dos problemas?

Um exemplo seria uma orientação alimentar para níveis elevados de colesterol. Às vezes tem-se a orientação substituída pela entrega de um impresso de dieta. Sabemos que isto só não basta, mas, algumas vezes, ficamos nela somente. Aquela pessoa terá que superar as restrições que estão sendo sugeridas pela dieta. Em algumas situações, contudo, lançamos mão de atenção mais cuidadosa, parando para ouvir as dificuldades daquela pessoa no que se refere a seguir as orientações escritas; outras vezes, orientamos uma adaptação gradativa, ou tentamos articular um atendimento pela psicologia, ou empregamos outro recurso qualquer para ajudá-la a superar as dificuldades.

Enfatizamos também nossa potencialidade de interferir nesse processo de determinação de necessidades de saúde, assumindo o papel de ator social na defesa de um projeto, na conformação de um dado modelo assistencial.

No que concerne àqueles com responsabilidade de gestão de serviços, parece ser preciso que olhem também para o trabalhador enquanto um ser tão carente quanto os usuários que são atendidos, estando sujeito às relações sociais da mesma forma; portanto, é essencial considerar este ponto na gerência dos recursos humanos, posto que os trabalhadores não são coisas, são seres humanos que têm vontades, desejos, agem e reagem a tudo e a todos, executando práticas de saúde que conformam um dado modelo assistencial, que pode não ser exatamente aquele que desejam ou que projetaram.

Quando se planejou o modelo que dividiu o trabalho do médico na rede pública em préconsulta, consulta e pós-consulta com o objetivo de racionalizar o uso do recurso mais caro do processo de produção em saúde - o trabalho do médico -, idealizou-se fazer a pré e a pós consulta, que previa orientação, educação em saúde, espaço de escuta e atenção a serem realizadas por um trabalhador treinado e de menor custo. No entanto, observa-se que essas atividades hoje parecem limitar-se a repetir, quando muito, a leitura da receita prescrita pelo médico, não acrescentado nada de novo ou de específico dessa outra prática profissional, descaracterizando a enfermagem até mesmo enquanto profissão.

Esse processo desemboca no não reconhecimento, pelo trabalhador, do outro como um homem rico de necessidades, além de não se reconhecer como sujeito de ação, possibilitando a perda da compreensão do processo saú- 
de-doença; assim, tanto o trabalhador como o usuário se transformam em fragmentos de um todo que não se recompõe.

Com isso queremos dizer que o trabalhador tem potência de trabalho, com certa autonomia em decidir "coisas" e fazer "coisas" que as práticas de planejamento e gerência em saúde não alcançam. Merhy (1997) e Merhy et al. (1997) denominam autogoverno essa autonomia, e é esse poder que faz com que o trabalhador faça uso (ou não) de todo o seu arsenal tecnológico no momento do atendimento do usuário, dependendo da forma como lhe "bater" internamente a relação.

Esse trabalhador é um homem, que está presente na relação de atendimento com toda sua individualidade, seus desejos, sentimentos, paixões, ódios, e nela vai objetivar sua subjetividade e objetividade manifestas naquela intercessão. A este fazer, a este certo modo de governar, de agir para a produção de bens, bens/produtos, bens/relações, Merhy (1997) e Merhy et al. (1997) denominam micropolítica do trabalho vivo em saúde.

Nestas relações, no encontro entre trabalhador e usuário, uma disputa de interesses entra em jogo, cujo resultado é impossível prever. Desta forma, não se pode dizer qual será o resultado a ser obtido nesta conjuntura: se satisfará a necessidade do usuário, do trabalhador e do serviço.

\section{Entendendo a prática de relações - a enfermagem na saúde coletiva}

No processo assistencial de enfermagem e, em particular, de enfermagem em saúde coletiva, o trabalhador lança mão tanto de uma série de tecnologias que incluem os equipamentos e o instrumental necessários ao desenvolvimento do trabalho - como, por exemplo, os aparelhos, a estrutura física, os procedimentos técnicos, os folhetos educativos, os conhecimentos estruturados acerca da epidemiologia, planejamento em saúde e outros -, quanto de tecnologias que envolvem as relações entre os sujeitos no processo de assistência, que dizem respeito à escuta, ao atendimento humanizado, ao vínculo e ao respeito pelo outro, enquanto alguém que porta uma dificuldade, um problema ou uma necessidade qualquer.

O trabalhador estará inserido em um contexto, executando seu trabalho em dada estruturação determinada pelo serviço, mas pode encontrar, nesse fazer, linhas de escape para dar outro desenho ao trajeto de solução do problema que não aquele tradicionalmente pre- visto para os casos “em geral”. Aqui está a chave dessas relações.

Os elementos mais fixos do trabalho - como as placas, os folhetos, as regras, as rotinas, as orientações e o próprio conhecimento - têm que ser levados em conta, pois são necessários à estruturação da relação de atendimento, mas o que se destaca é a forma como se os utiliza, representando preocupação para com o usuário. Para exemplificar, quando se trata da sinalização da unidade de saúde, informa-se a localização das salas e setores de atendimento por meio de placas, cartazes, painéis informativos e outros. Desde a entrada, tais indicações não são destacadas de maneira suficiente, as placas são pouco evidentes e os setores, em muitas situações, ficam sem ninguém para informar. Não se está dizendo que toda a sinalização do local deva ser mudada, mas, sim, que o serviço como um todo, através de seus trabalhadores, deva considerar a sinalização tal como se encontra e orientar os usuários segundo suas necessidades, ficando assim atentos em identificar, dentre o conjunto de usuários, aqueles que apresentam dificuldades, no momento, para localizar-se espacialmente na unidade de saúde, seja por limitação de escolaridade, seja por dificuldades circunstanciais, como dor, ansiedade, medo; este é um exercício constante da eqüidade e acessibilidade.

É essencial entender que, nas relações, os envolvidos são importantes um para o outro, pois nelas um não existe sem o outro. Há necessidade desse respeito mútuo, no qual se abre a disponibilidade de escuta, de destinar-se um espaço-tempo interno para a relação, em que se pode acolher o outro em seu momento de dificuldade, que não é só biológica; muito pelo contrário, a cada dia, outros fatores tornam-se preponderantes na determinação dos sofrimentos humanos que os meramente envolvidos com as dores físicas. Esta disponibilidade também deve abrir-se para o responsabilizar-se pela ação de saúde, pelo cuidar, por aumentar o coeficiente de autonomia do usuário (Campos, 1994).

O trabalho em saúde coletiva e em enfermagem precisa ser tomado de forma mais leve... como nos propõe Calvino (1991), utilizando o mito de Perseu. Este, para decepar a cabeça da Medusa sem se deixar petrificar, não volta jamais o olhar para a face da Górgona, mas apenas para a imagem que vê refletida em seu escudo de bronze. Voa com sandálias aladas e se sustenta no que há de mais leve, as nuvens e o vento...

Matumoto (1998) utiliza-se desta alegoria para pensar em dois aspectos do trabalho em 
saúde que são extremamente pertinentes tanto para a saúde coletiva como para a enfermagem.

" 1 - O trabalho em saúde não precisa ser condenado ao peso da dor, do sofrimento, das carências. É preciso inventar cada um à sua maneira, formas como as de Perseu, de voar para outros espaços de produção de ações de saúde.

2 - As ações em saúde requerem ser operadas através da leveza que pode ser obtida por artefatos, como o escudo de Perseu. Olhar diretamente para os problemas pode nos petrificar, não permitindo que alcancemos seu âmago. [... necessitamos ...] meios para não nos transformarmos em pedras e que podem nos possibilitar formas de efetivar a equidade, acessibilidade e integralidade da assistência" (Matumoto, 1998:203).

O desafio está posto: abrirmo-nos enquanto enfermagem e saúde coletiva, assumindo nosso lugar social como sujeitos da ação para uma nova prática de relações, incluindo tecnologias tais como o vínculo, o acolhimento, a autonomização, o estar disponível para as relações. Correr o risco de voar e descobrir novos horizontes.

\section{Referências}

ALMEIDA, M. C. P.; MISHIMA, S. M. \& PEDUZZI, M., 1999. A pesquisa em enfermagem fundamentada no processo de trabalho: Em busca da compreensão e qualificação da prática de enfermagem. Ribeirão Preto: Escola de Enfermagem de Ribeirão Preto, Universidade de São Paulo. (mimeo.)

ALMEIDA, M. C. P.; SILVA, G. B.; RUFFINO, M. C.; GOMES, D. S. \& YAZLE-ROCHA, J. S., 1981. Contribuição ao Estudo da Prática de Enfermagem. Ribeirão Preto: Escola de Enfermagem de Ribeirão Preto, Universidade de São Paulo. (mimeo.)

ALMEIDA FILHO, N., 1997. Transdisciplinaridade e saúde coletiva. Ciência \& Saúde Coletiva, 2:5-20.

CALVINO, I., 1991. Seis Propostas para o Próximo Milênio. São Paulo: Editora Companhia das Letras.

CAMPOS, G. W. S., 1994. Considerações sobre a arte e a ciência da mudança. O caso da saúde. In: Inventando a Mudança na Saúde (L. C. O. Cecílio, org.), pp. 29-87, São Paulo: Editora Hucitec.

CAMPOS, G. W. S., 1997. Subjetividade e administração de pessoal. In: Agir em Saúde. Um Desafio para o Público (E.E. Merhy \& R. Onocko, org.), pp. 229-266, São Paulo: Editora Hucitec.

CANESQUI, A. M.,1995. As ciências sociais, a saúde e a saúde coletiva. In: Dilemas e Desafios das Ciências Sociais na Saúde Coletiva (A. M. Canesqui, org.), pp. 19-35, Rio de Janeiro: ABRASCO/São Paulo: Hucitec.
COCCO, M. I., 1997. Restruturação Produtiva e o Setor Saúde: Trabalhadores de Enfermagem em Saúde. Tese de Doutorado, Ribeirão Preto: Escola de Enfermagem de Ribeirão Preto, Universidade de São Paulo.

CORBISHLEY, A. C. M., 1998. O Trabalho de Enfermagem no Processo de Construção de um Modelo Assistencial em Saúde Coletiva. Tese de Doutorado, Rio de Janeiro: Escola de Enfermagem Anna Nery, Universidade Federal do Rio de Janeiro.

EGRY, E. Y., 1996. Saúde Coletiva: Construindo um Novo Método em Enfermagem. São Paulo: Editora Ícone.

FELIPE, J. S., 1989. Uma avaliação da produção teórico-científica da medicina social no Brasil. Saúde em Debate, 27:59-66.

FERREIRA, S. L., 1994. A Mulher e os Serviços Públicos de Saúde: Análise do Processo de Trabalho em Unidades Básicas de Salvador (BA). Tese de Doutorado, Ribeirão Preto: Escola de Enfermagem de Ribeirão Preto, Universidade de São Paulo.

FORTUNA, C. M., 1999. O Trabalho de Equipe numa Unidade Básica de Saúde: Produzindo e Re-produzindo-se em Subjetividades. Em Busca do Desejo, do Devir e de Singularidades. Dissertação de Mestrado, Ribeirão Preto: Escola de Enfermagem de Ribeirão Preto, Universidade de São Paulo.

GERMANO, R. M., 1983. Educação e Ideologia da Enfermagem no Brasil. São Paulo: Editora Cortez. 
GOMES, R. \& DESLANDES, S. F., 1994. Interdisciplinaridade na saúde pública: Um campo em construção. Revista Latino-Americana de Enfermagem, 2:103-114.

LANA, F. C. F., 1992. Organização Tecnológica do Trabalho em Hanseníase com Introdução da Poliquimioterapia. Dissertação de Mestrado, Ribeirão Preto: Escola de Enfermagem de Ribeirão Preto, Universidade de São Paulo.

MATUMOTO, S., 1998. O Acolhimento: Um Estudo sobre seus Componentes e sua Produção em uma Unidade da Rede Básica dos Serviços de Saúde. Dissertação de Mestrado, Ribeirão Preto: Escola de Enfermagem de Ribeirão Preto, Universidade de São Paulo.

MENDES-GONÇALVES, R. B., 1992. Práticas de Saúde: Processos de Trabalho e Necessidades. São Paulo: Prefeitura Municipal de São Paulo.

MERHY, E. E., 1985. O Capitalismo e a Saúde Pública. São Paulo: Papirus.

MERHY, E. E., 1997. Em busca do tempo perdido: A micropolítica do trabalho vivo em saúde. In: Agir em Saúde. Um Desafio para o Público (E. E. Merhy \& R. Onocko, org.), pp. 71-112, São Paulo: Editora Hucitec.

MERHY, E. E.; CHAKKOUR, M.; STÉFANO, E.; SANTOS, C. M.; RODRIGUES, R. A. \& OLIVEIRA, P. C. P., 1997. Em busca de ferramentas analisadoras das tecnologias em saúde. A informação e o dia a dia de um serviço, interrogando e gerindo trabalho em saúde. In: Agir em Saúde. Um Desafio para o Público (E. E. Merhy \& R. Onocko, org.), pp. 113150, São Paulo: Editora Hucitec.

MISHIMA, S. M., 1995. Constituição do Gerenciamento Local na Rede Básica de Saúde em Ribeirão Preto. Tese de Doutorado, Ribeirão Preto: Escola de Enfermagem de Ribeirão Preto, Universidade de São Paulo.
NUNES, E. D., 1994. Saúde coletiva: História de uma idéia e de um conceito. Saúde e Sociedade, 3:5-21.

PEDUZZI, M., 1999. Equipe Multiprofissional de Saúde: A Interface entre Trabalho e Interação. Tese de Doutorado, Campinas: Faculdade de Ciências Médicas, Universidade de Campinas.

PEREIRA, J. C., 1986. Medicina, saúde e sociedade. Estudos de Saúde Coletiva, 4:29-37.

PIRES FILHO, F. M., 1987. O que é saúde pública? Cadernos de Saúde Pública, 3:62-70.

SCHRAIBER, L. B., 1996. Ciência, Trabalho e trabalhador em saúde: Contribuições de Ricardo Bruno Mendes Gonçalves para a compreensão da articulação entre saber, prática e recursos humanos. Divulgação em Saúde, 14:7-12.

SILVA, E. M.,1997. A Supervisão do Trabalho de Enfermagem em Saúde Pública no Nível Local. Tese de Doutorado, Ribeirão Preto: Escola de Enfermagem de Ribeirão Preto, Universidade de São Paulo.

SILVA, G. B., 1986. Enfermagem Profissional. Análise Crítica. São Paulo: Editora Cortez.

TESTA, M., 1995. Pensamento Estratégico e Lógica da Programação. O Caso da Saúde. São Paulo: Editora Hucitec.

VAZ, M. R. C.,1992. Conceito e Prática de Saúde: Adequação no Trabalho de Controle da Tuberculose. Tese de Doutorado, Florianópolis: Departamento de Enfermagem, Universidade Federal de Santa Catarina.

VILLA, T. C. S.,1992. A Enfermeira nos Serviços de Saúde Pública no Estado de São Paulo (1967-1983). Tese de Doutorado, Ribeirão Preto: Escola de Enfermagem de Ribeirão Preto, Universidade de São Paulo. 